Psychotherapeut 2014 · 59:185-186

DOI 10.1007/s00278-014-1049-1

Online publiziert: 19. April 2014

(c) Springer-Verlag Berlin Heidelberg 2014

Hansruedi Ambühl

Psychotherapeutische Praxis, Bern

\title{
Standortbestimmung zur Methodenintegration in der Psychotherapie
}

higen und selbstbestimmten Klienten zu tun. Eine dritte Quelle von Unterschieden kann schließlich auf Unterschiede in den Überzeugungen, Wertvorstellungen und Persönlichkeitsstrukturen der Psychotherapeuten selbst zurückgeführt werden. Einige Therapeuten sind von Natur aus eher kontemplativ, während andere stärker handlungsorientiert sind; einige gehen eher rational und planmäßig, andere intuitiv vor; einige erscheinen eher extravertiert, andere hingegen eher zurückgezogen usw.

\section{„Neue Kulturen schaffen"}

Seit dem Erscheinen des Buchs von Grawe et al. (1994) Psychotherapie im Wandel - Von der Konfession zur Profession hat sich in der deutschsprachigen Psychotherapielandschaft so einiges bewegt. Grawes durchaus auch polemisch gemeinte Aussagen führten zunächst dazu, dass sich die Vertreter der einzelnen Therapierichtungen vermehrt darum bemühten, die Effektivität ihrer Methoden mithilfe wissenschaftlicher Studien zu belegen, was insgesamt zu einem seriöseren Ansehen der Psychotherapie führte. Andererseits traten anstelle des unsäglichen Glaubenskriegs zwischen den Therapieschulen vermehrt gegenseitiger Respekt voreinander und das Bemühen um einen gemeinsamen Dialog in den Vordergrund. Die Suche nach der einzig wahren und richtigen Psychotherapie wich zunehmend der Einsicht, dass sich das therapeutische Vorgehen von Fall zu Fall nach den unterschiedlichen Gegebenheiten des jeweiligen Patienten zu richten habe. In gewisser Wei- se bedeutet dies auch eine Würdigung der Gründerväter der Therapieschulen, allerdings mit dem Unterschied, dass es im Einzelfall nicht mehr um ein „Entweder-oder“, sondern um ein "Sowohl-alsauch" geht. So betrachtet hat in den letzten 20 Jahren ein deutlich spürbarer Kulturwandel stattgefunden. Die Frage, wie genau sich dieser Wandel in der heutigen psychotherapeutischen Praxis manifestiert, war Thema der Lindauer Psychotherapiewochen 2013 im Rahmen des Leitthemas „Neue Kulturen schaffen“. Es fand ein Vorlesungszyklus statt, der so aufgebaut war, dass Vertreter der beiden psychotherapeutischen Verfahren „psychodynamische Therapien“ und „Verhaltenstherapie" darüber referierten, welche Bedeutung zentrale Bestandteile der jeweils anderen Therapierichtung für die eigene therapeutische Arbeit haben. Also: Welche Bedeutung haben Kognitionen und kognitive Techniken in psychodynamischen Therapien? Wie werden Konflikte in der Verhaltenstherapie bearbeitet? Welchen Stellenwert haben Exposition und Psychoedukation in psychodynamischen Therapien? Welche Rolle spielen Übertragung und Gegenübertragung in der Verhaltenstherapie? Am Schluss dieser Vortragsreihe fand eine Podiumsdiskussion statt, in der bezüglich des aktuellen Stands der Psychotherapie von den Referenten und den Teilnehmern ein derzeitiges Fazit gezogen wurde.

In diesem Themenheft finden sich die überarbeiteten Vorträge dieses Vorlesungszyklus. Luise Reddemann stellt in ihrem Beitrag zur Arbeit an Kognitionen in psychodynamischen Therapien zu- gel mit hochgebildeten, introspektionsfä- 
nächst dar, was man unter Kognitionen in der Verhaltenstherapie versteht und welche Rolle kognitive Konzepte in ganz unterschiedlichen Therapieformen spielen. Sie vergleicht die kognitive Arbeit mit neuen Landkarten, in denen die Landvermesser (Gründer anderer Therapierichtungen) wohl Verschiedenes festgehalten haben, was auch für andere hilfreich sein kann, um ein bestimmtes Ziel zu erreichen. Anhand verschiedener Fallbeispiele aus ihrer Praxis kommt sie zu dem Schluss, dass der kognitive Zugang im Sinne der Ermutigung zur Verhaltensbeobachtung und der dadurch möglichen Veränderung kognitiver Verzerrungen oft die erfolgversprechende Grundlage für einsichtsorientierte therapeutische Arbeit darstellt.

Franz Caspar geht in seinem Beitrag zum Stellenwert der Arbeit an intrapersonalen Konflikten nicht auf bestehende psychodynamische Konzepte ein, sondern weist darauf hin, dass es bereits im Rahmen der Lerntheorien und der klassischen Verhaltenstherapie Ansätze für die Arbeit an Konflikten gegeben habe. Diese eher rudimentären Konzepte wurden in der modernen kognitiven Verhaltenstherapie maßgeblich durch die Entwicklung der plananalytischen Fallkonzeption von Grawe u. Caspar (1984) verbessert, die eine klare Ortung intrapsychischer Konflikte ermöglicht und der Bearbeitung zugänglich macht. Anhand von Grawes Konsistenztheorie wird schließlich verdeutlicht, dass Konflikte zwischen wichtigen Motiven der Realisierung von Bedürfnissen im Weg stehen und hoch mit Psychopathologie korrelieren.

Katharina Parisius und Ulrich Sachsse beschreiben Psychoedukation und Exposition als Elemente der Verhaltenstherapie und fragen sich, wie sich diese innerhalb eines psychodynamischen Behandlungsspektrums schlüssig begründen lassen. Mit Bezug auf Küchenhoff (2009) halten sie für eine konzeptuelle Integration unterschiedlicher Verfahren eine Metatheorie für erforderlich, die Behandlungselemente nicht einfach regellos aneinanderreiht, sondern neue ganzheitliche Konzepte höherer Komplexität liefert. Am Beispiel der Behandlung von Frauen mit komplexen Traumafolgestörungen weisen sie darauf hin, dass sich die traumazent- rierte Therapie bereits auf dem Weg zur Formulierung einer Metatheorie für eine konzeptuelle Integration befinde.

Eva Bänninger-Huber nimmt die therapeutische Beziehung genauer unter die Lupe und beschreibt zunächst die historische Entwicklung von „Übertragung“ und „Gegenübertragung“ in der Psychoanalyse. Dann zeigt sie auf, wie die moderne Verhaltenstherapie Anleihen aus der "control mastery theory“ von Weiss (1986) macht und die Beziehung auch als Lernfeld betrachtet, die es dem Patienten ermöglichen soll, seine aufgrund traumatischer Kindheitserfahrungen entwickelten pathogenen Überzeugungen zu überwinden. Schließlich zeigt sie anhand der Analyse prototypischer affektiver Mikrosequenzen auf, wie Übertragungsphänomene heute in der Affektforschung präziser identifiziert werden können.

\section{Korrespondenzadresse}

Dr. phil. Hansruedi Ambühl

Psychotherapeutische Praxis

Aarbergergasse 46, 3011 Bern

Schweiz

hansruedi.ambuehl@sunrise.ch

\section{Einhaltung ethischer Richtlinien}

Interessenkonflikt. Hansruedi Ambühl gibt an, dass kein Interessenkonflikt besteht.

\section{Literatur}

Grawe K, Bernauer R, Donati R (1984) Psychotherapie im Wandel. Von der Konfession zur Profession. Hogrefe, Göttingen

Grawe K, Caspar F (1984) Die Plananalyse als Konzept und Instrument für die Psychotherapieforschung. In: Baumann U (Hrsg) Psychotherapie. Macro/Microperspektive. Hogrefe, Göttingen, S 177-199

Küchenhoff J (2009) Der integrative Prozess in der Psychotherapie: Methodenvielfalt - Synergismus - Integration. Schweiz Arch Neurol Psychiatr 160:12-19

Orlinsky DE (1994) „Learning from many masters". Ansätze zu einer wissenschaftlichen Integration psychotherapeutischer Behandlungsmodelle. Psychotherapeut 39:2-9

Weiss J (1986) Part I: theory and clinical observations. In: Weiss J, Sampson H; The Mount Zion Psychotherapy Research Group (Hrsg) The psychoanalytic process: theory, clinical observation, and empirical research. Guilford, New York, S 3-138

\section{Schwerpunktthemen}

Das Herausgebergremium der Zeitschrift Psychotherapeut lädt Autorinnen und Autoren ein, an den geplanten Schwerpunkten mitzuarbeiten und geeignete Manuskripte einzureichen. Diese werden dem üblichen Reviewverfahren unterzogen. Darüber hinaus freuen wir uns über die Zusendung freier (unaufgeforderter) Originalia zu selbstgewählten Themen.

Bitte schicken Sie Ihren Beitrag an die Redaktion:

Regine.Karcher-Reiners@springer.com

Die Schwerpunktplanung ist vorläufig und kann kurzfristig umdisponiert werden.

Heft 6/14: DSM-5/ICD-11

(Deadline 30.06.2014)

Heft 1/15: Die Zukunft der

Psychotherapie

(Deadline 30.08.2014)

Heft 2/15: LPW

(Deadline 30.10.2014)

Heft 3/15: Der psychotherapeutische

Prozess

(Deadline 30.12.2014)

Heft 4/15: Selbstbeschädigung und Symptomatik autoaggressiven Verhaltens (Deadline 30.02.2015) 